\title{
Extent of linkage disequilibrium, consistency of gametic phase, and imputation accuracy within and across Canadian dairy breeds
}

\author{
S. G. Larmer, ${ }^{\star 1}$ M. Sargolzaei, ${ }^{\star} \dagger$ and F. S. Schenkel ${ }^{\star}$ \\ ${ }^{*}$ Centre for Genetic Improvement of Livestock, Animal and Poultry Science Department, University of Guelph, Guelph, Ontario, N1G 2W1 Canada \\ †L'Alliance Boviteq, Saint-Hyacinthe, Quebec, J2T 5H1, Canada
}

\begin{abstract}
Genomic selection requires a large reference population to accurately estimate single nucleotide polymorphism (SNP) effects. In some Canadian dairy breeds, the available reference populations are not large enough for accurate estimation of SNP effects for traits of interest. If marker phase is highly consistent across multiple breeds, it is theoretically possible to increase the accuracy of genomic prediction for one or all breeds by pooling several breeds into a common reference population. This study investigated the extent of linkage disequilibrium (LD) in 5 major dairy breeds using a 50,000 (50K) SNP panel and 3 of the same breeds using the 777,000 (777K) SNP panel. Correlation of pair-wise SNP phase was also investigated on both panels. The level of LD was measured using the squared correlation of alleles at 2 loci $\left(\mathrm{r}^{2}\right)$, and the consistency of SNP gametic phases was correlated using the signed square root of these values. Because of the high cost of the $777 \mathrm{~K}$ panel, the accuracy of imputation from lower density marker panels $[6,000(6 \mathrm{~K})$ or $50 \mathrm{~K}]$ was examined both within breed and using a multi-breed reference population in Holstein, Ayrshire, and Guernsey. Imputation was carried out using FImpute V2.2 and Beagle 3.3.2 software. Imputation accuracies were then calculated as both the proportion of correct SNP filled in (concordance rate) and allelic $\mathrm{R}^{2}$. Computation time was also explored to determine the efficiency of the different algorithms for imputation. Analysis showed that $L D$ values $>0.2$ were found in all breeds at distances at or shorter than the average adjacent pair-wise distance between SNP on the $50 \mathrm{~K}$ panel. Correlations of r-values, however, did not reach high levels $(<0.9)$ at these distances. High correlation values of SNP phase between breeds were observed $(>0.94)$ when the average pair-wise distances using the $777 \mathrm{~K}$ SNP panel were examined. High concordance rate (0.968-0.995) and allelic $\mathrm{R}^{2}(0.946-0.991)$ were found for all breeds when imputation was carried
\end{abstract}

Received March 18, 2013

Accepted January 14, 2014.

${ }^{1}$ Corresponding author: slarmer@uoguelph.ca out with FImpute from 50K to 777K. Imputation accuracy for Guernsey and Ayrshire was slightly lower when using the imputation method in Beagle. Computing time was significantly greater when using Beagle software, with all comparable procedures being 9 to 13 times less efficient, in terms of time, compared with FImpute. These findings suggest that use of a multibreed reference population might increase prediction accuracy using the $777 \mathrm{~K}$ SNP panel and that $777 \mathrm{~K}$ genotypes can be efficiently and effectively imputed using the lower density 50K SNP panel.

Key words: linkage disequilibrium, dairy, imputation

\section{INTRODUCTION}

The advent of genomic selection has been a major breakthrough in the breeding programs of many dairy breeds, and it continues to improve with the advent of new technologies to increase the accuracy of selection and the reliability of the methods used. To begin genomic selection within a breed or group of breeds, an important step is assessing the level of linkage disequilibrium (LD; Meuwissen et al., 2001). Linkage disequilibrium is a measure of the nonrandom association of alleles that helps us to infer the alleles present at other loci, especially at QTL that have an effect on phenotypes of interest. Numerous studies have found high levels of LD between adjacent marker pairs as well as LD extending over tens of centimorgans (Farnir et al., 2000). However, useful LD in Holsteins was only found at distances $<100 \mathrm{~kb}$ (Sargolzaei et al., 2008). Useful LD was defined as an $\mathrm{r}^{2}$ value $>0.3$, which is the level of LD deemed necessary for successful association studies (Ardlie et al., 2002). Calus et al. (2008) showed, however, that accuracy of genomic selection can be implemented accurately when the level of LD, based on $r^{2}$, is $>0.2$.

The 2 factors that affect accuracy of genomic selection that can be controlled are the level of LD between markers and QTL and the size of the reference population of animals used to estimate SNP effects on phenotypes (Hayes et al., 2009). The level of LD between markers and QTL can be controlled by utilizing very dense mark- 
er panels. Genomic selection, as has been implemented in the Holstein, Jersey, and Brown Swiss populations in North America, uses the 50,000 marker (50K) panel. With the advent of SNP panels with up to 777,000 markers $(\mathbf{7 7 7} \mathbf{K})$, the accuracy of genomic selection can be increased. Calus et al. (2008) found, using simulated data, that if $\mathrm{r}^{2}$ is increased from 0.1 to 0.2 , the accuracy of genomic selection increased from 0.68 to 0.82 . Brito et al. (2011) reported that for similar accuracy of genomic selection, approximately half as many animals would be needed in the training population when high-density panels were used. Erbe et al. (2012) showed slight gains in genomic EBV (GEBV) accuracy when using a highdensity marker panel $(\sim 800,000)$ compared with using the $50 \mathrm{~K}$ panel in Holstein and Jersey populations. This increase in accuracy is relatively small because enough linkage within a breed to effectively implement genomic selection can be found on the $50 \mathrm{~K}$ panel, especially with large reference populations of genotyped animals, such as is available in the Holstein breed. For a population with fewer animals genotyped $(\mathrm{n}=540)$, Erbe et al. (2012) also showed that a combined reference population could increase GEBV accuracy by an average of $4 \%$ for various production traits when using a high-density marker panel.

These dense marker panels, however, are expensive and can be a major limitation to the number of animals genotyped. The advent of imputation has significantly aided this problem, making it possible for many animals to be genotyped with lower-density marker panels and imputed to higher density for genomic selection. In Canada, imputation takes place regularly from approximately $3,000(\mathbf{3 K})$ and $6,000(\mathbf{6 K}) \mathrm{SNP}$ to the $50 \mathrm{~K}$ SNP panel by using the FImpute program (Sargolzaei et al., 2011a) and both the family and population algorithms. For a large reference population, imputation from $\sim 3 \mathrm{~K}$ to $\sim 50 \mathrm{~K}$ has been shown to be accurate using both family-based and population-based methods, with accuracy exceeding 0.90 . When family information is available, family-based methods do have an advantage over population-based methods (Zhang and Druet, 2010).

Once the level of LD between marker and causative mutation has been maximized by using the highest density panel available or with full sequence, the reference population must be grown to increase the accuracy of genomic prediction. Luan et al. (2009) showed an increase in accuracy of approximately $5 \%$ for genomic selection of production traits in Norwegian Red cattle when the reference population grew from 250 to 400 animals. Liu et al. (2011) also saw an increase in the variance of SNP effects as the reference population size grew, allowing for larger SNP effects to be detected and selected upon. One way to increase the training population size for genomic selection would be to use a breed with more genotyped individuals in the reference population for a breed with fewer genotyped animals. It was found that for Holsteins and Jerseys, one breed could not be directly used as the sole training population for selection in the other breed using the $50 \mathrm{~K}$ marker panel, but results were more promising when both breeds were combined into a common population, although gains were modest (Pryce et al., 2011). For genomic selection to be effective, markers and QTL need to be in the same linkage phase across the populations, and linkage phase must be consistent from the training population to the validation population. de Roos et al. (2008) estimated that for breeds to be pooled into a common reference population that increases accuracy of genomic prediction, approximately 300,000 evenly spaced markers would be needed to ensure adequate consistency of phase across breeds in the multi-breed reference population for genomic selection.

The goal of this study was to explore the extent of LD in 5 Canadian dairy breeds using the 50K SNP panel, as well as that in 3 breeds using the $777 \mathrm{~K}$ SNP panel. Consistency of phase was then explored to determine the possible use of a multi-breed reference population for genomic selection in Ayrshires and Guernseys using both the $50 \mathrm{~K}$ and $777 \mathrm{~K}$ SNP panels. Accuracy of imputation from $6 \mathrm{~K}$ or $50 \mathrm{~K}$ to $777 \mathrm{~K}$ using real Ayrshire, Guernsey, and Holstein data was then calculated. Both a population-based (Beagle 3.3.2; Browning and Browning, 2007) and family and population-based (FImpute v2.2; Sargolzaei et al., 2011a) method were explored. The effects of a multi-breed reference population and the effect of having direct ancestors genotyped were also examined. If phase consistency is high, multi-breed imputation is expected to increase accuracy for populations with few genotyped individuals. In addition, imputation from $6 \mathrm{~K}$ to $777 \mathrm{~K}$ using a 2 -step approach was assessed in the Holstein breed.

\section{MATERIALS AND METHODS}

\section{Data}

Genotypes from Holstein ( $\mathrm{n}=47,433)$ Jersey ( $\mathrm{n}=$ $4,517)$, and Brown Swiss $(\mathrm{n}=1,566)$ were taken on animals from the North American Collaboration on Genomic Prediction, many of which were proven sires. Genotyping was performed using the Illumina $50 \mathrm{~K}$ BeadChip (Illumina Inc., San Diego, CA). In addition, 476 proven Ayrshire bulls and 61 proven Guernsey bulls were genotyped with the BovineHD $(777 \mathrm{~K})$ panel (Illumina Inc.). Holstein $777 \mathrm{~K}$ genotypes $(\mathrm{n}=1,115)$ were also obtained from The North American Collaboration on Genomic Prediction. 
For the analyses of the extent of $\mathrm{LD}$ and gametic phase consistency, SNP were filtered for minor allele frequencies $<0.05$ to assess only SNP that were segregating in a significant number of animals in the population. Single nucleotide polymorphisms were also excluded if the missing SNP call frequency for that SNP locus was greater than 10\%. The SNP present on the $\mathrm{X}$ chromosome as well as any SNP with unknown map position were also excluded for the purposes of this study.

The 50K SNP panel was used as a reference to filter SNP from the 777K panel for Ayrshire and Guernsey breeds, saving only SNP that were present on both panels to ensure consistency of phase comparisons across all 5 breeds at the lower density. This resulted in 39,127 (out of 43,382) SNP being considered that were consistent between the $50 \mathrm{~K}$ and $777 \mathrm{~K}$ panels. For the 3 breeds genotyped on the high-density panel, markers were also filtered to a subset of only the SNP contained on the $6 \mathrm{~K}$ panel. This resulted in a subset of 6,556 markers from the $777 \mathrm{~K}$ panel available for imputation from $6 \mathrm{~K}$ to $777 \mathrm{~K}$. For the purpose of imputation, SNP were not filtered for minor allele frequency as they were for determining extent of $\mathrm{LD}$ and gametic phase consistency, so that a larger subset was available from the $50 \mathrm{~K}$ panel, resulting in 39,943 markers available for imputation from $50 \mathrm{~K}$ to $777 \mathrm{~K}$. It should also be noted that all 6,556 markers on the $6 \mathrm{~K}$ panel were a part of the 39,943 markers on the $50 \mathrm{~K}$ panel.

To perform imputation, animals with $777 \mathrm{~K}$ genotypes were divided up into 2 distinct groups: reference and imputation animals. This sorting was done by birth year in an effort to capture as many sires of imputation animals in the reference population as possible, creating a practical scenario that mimics how imputation is carried out for routine genomic evaluations. In Ayrshire and Guernsey breeds, animals born in 2000 or later were included in the imputation population. This gave reference populations of 313 and 42 and imputation groups of 163 and 19 for Ayrshire and Guernsey, respectively. Holsteins born in 2004 or later were included in the imputation group, creating populations of 892 and 223 for reference and imputation. Several Holsteins had no known birthdate and these were included in the reference population. The number of imputed animals who have a genotyped sire, dam, maternal grandsire, and paternal grandsire was also examined.

\section{Extent of Linkage Disequilibrium}

Reconstructing haplotypes accurately requires a large sample size, a high degree of relatedness between animals, or both. Due to small population sizes and the small degree of relatedness between animals in the genotyped populations, haplotypes could not be accurately reconstructed to determine the extent of $\mathrm{LD}$ in all breeds, only for Holstein. Haplotypes for Holsteins were reconstructed as in Sargolzaei et al. (2008), and $\mathrm{LD}$ results were compared with the same scenarios when haplotypes were not reconstructed. In this case, very little difference was seen between the 2 scenarios. For this reason, Holstein haplotypes were not reconstructed so as to allow consistent comparisons across breeds.

Linkage disequilibrium was determined using $\mathrm{r}^{2}$, which is the squared correlation of alleles at 2 loci. This was calculated between pairs of loci on each chromosome to determine the LD at close distances as well as the LD decay over distance. The $\mathrm{r}^{2}$ values were determined for the $50 \mathrm{~K}$ panel for all 5 breeds and for the $777 \mathrm{~K}$ panel for Holstein, Ayrshire, and Guernsey breeds. For computational ease, $\mathrm{r}^{2}$ was determined only at distances $<0.5 \mathrm{Mb}$ with the $777 \mathrm{~K}$ panel, as the $50 \mathrm{~K}$ panel gives an accurate measure of LD decay beyond this distance. The $\mathrm{r}^{2}$ value was calculated as follows:

$$
r^{2}=\frac{D^{2}}{f(A) f(a) f(B) f(b)},
$$

where $D=f(A B)-f(A) f(B)$, and $f(A B), f(A), f(a)$, $f(B)$, and $f(b)$, are observed frequencies of haplotype $\mathrm{AB}$ and alleles A, a, B, and b, respectively. When haplotypes are not reconstructed, an unbiased estimator of $D$, under assumption that coupling and repulsion heterozygotes are equally likely, is

$$
\begin{aligned}
& D=\frac{N}{N-1} \times \\
& {\left[\frac{4 N_{A A B B}+2\left(N_{A A B b}+N_{A a B B}\right)+N_{A a B b}}{2 N}\right]-2 \times f(A) \times f(B)}
\end{aligned}
$$

(Lynch and Walsh, 1998), where $N$ is the total number of individuals, and $N_{A A B B}, N_{A A B b}, N_{A a B B}$, and $N_{A a B b}$ are the corresponding number of individuals in each genotypic category (AABB, $\mathrm{AABb}, \mathrm{AaBB}$, and $\mathrm{AaBb}$ ). Not reconstructing haplotypes may lead to downward bias in the level of $\mathrm{LD}$, as the above estimator of $\mathrm{D}$ does not discriminate between coupling and repulsion heterozygotes, which may generally lead to lower absolute $D$ values. Therefore, the measure of $\mathrm{LD}$ in this study is expected to be conservative, if any bias exists. In Holsteins, as previously mentioned, the preliminary use of $D$ estimated from reconstructed haplotypes or the use of the approximated $D$ did not cause significant difference in the estimated $r^{2}$.

The $\mathrm{r}^{2}$ measure of LD was used because it is less biased by sample size and low allele frequency compared 
with other popular measures of LD, such as $D^{\prime}$ (Ardlie et al., 2002), although no LD measure is completely independent of allele frequency. Data were sorted into groups based on pair-wise marker distance to determine the breakdown of LD in each breed as the distance between markers increased.

\section{Consistency of Phase}

For each marker pair with a measure of $\mathrm{r}^{2}$, the signed $r$ value was determined by taking the square root of the $\mathrm{r}^{2}$ value and assigning the appropriate sign based on the calculated $D$ value. Data were sorted into groups based on pair-wise marker distance to determine the breakdown in correlation across distance and to be able to assess the correlation of signed $r$ values at the smallest distances possible given the number of SNP that were investigated. The $\mathrm{r}$ values were then correlated between breeds using PROC CORR in SAS (SAS Institute Inc., Cary, NC). This allows for the measurement of the breakdown in correlation of LD phase between breeds as distance between marker pairs increases. Correlations were calculated between all pairs of breeds available on a given SNP panel. This gave 10 breed pairs on the $50 \mathrm{~K}$ panel and 3 pairs on the $777 \mathrm{~K}$ panel, those 3 being Ayrshire-Guernsey, Ayrshire-Holstein, and Holstein-Guernsey.

\section{Imputation Scenarios}

Several imputation scenarios were carried out in this study. The primary goal was to evaluate the effect of family- and population-based versus population-based imputation both within breed and in a multi-breed reference population using the $777 \mathrm{~K}$ SNP panel. This was done by imputing all breeds individually as well as combining all populations for imputation both in Beagle (population-based; Browning and Browning, 2007) and in FImpute (family- and population-based; Sargolzaei et al., 2011a). Accuracy of imputation by the population-based algorithms of Beagle and FImpute was also examined to determine which software could impute more accurately in the absence of pedigree information, as is always the case for Beagle, which does not directly use family information. All the above scenarios were carried out only for the 50K SNP panel. Imputation accuracy for the $6 \mathrm{~K}$ panel was determined either as a single step (from $6 \mathrm{~K}$ to $777 \mathrm{~K}$ ) in all breeds or using a 2-step procedure (imputing from $3 \mathrm{~K}$ to $50 \mathrm{~K}$ followed by imputation from the imputed $50 \mathrm{~K}$ panel to the $777 \mathrm{~K}$ panel) in Holsteins. This was carried out both within breed and with a multi-breed reference, using only FImpute with family information included. In the Holstein population, many more 50K genotypes are available, so it was used to assess the 2-step procedure. For routine imputation to higher densities, these genotypes would be used in the first step of a 2-step imputation process. Accuracy of imputation with a larger reference population in the first step was examined by adding, 2,000, 4,000, and 6,000 randomly selected 50K Holstein genotypes. For all scenarios for the Ayrshire breed, using both imputation programs, the effect of having family members in the genotyped reference population was examined. Imputation accuracy rates were compared between animals who had genotyped sires or maternal grandsires in the reference population and those that had no close ancestors in the genotyped reference population.

\section{Calculation of Imputation Accuracy}

For this study, imputation accuracy was measured using 2 metrics: the ratio of correct call to overall call rate (concordance rate) and the allelic $\mathrm{R}^{2}$. It should also be noted that the most probable call was always assigned at a given locus, leaving no missing calls. Markers were filtered from the original $777 \mathrm{~K}$ genotype panel; thus, to calculate the correct call rate, the imputed genotypes were compared with the actual $777 \mathrm{~K}$ genotypes and the amount of correct calls was calculated. Correct calls are, therefore, those in which the call after imputation exactly matches that of the original $777 \mathrm{~K}$ genotype, excluding those markers that were present on the low-density marker panel. Allelic $\mathrm{R}^{2}$ was calculated as described by Browning and Browning (2009); that is, the squared correlation between the number of a given allele present in the true and imputed genotypes. The $\mathrm{R}^{2}$ values is expected to deviate more, being lower than the concordance rate, when the homozygous genotypes are more likely; that is, with low minor allele frequency (Ma et al., 2013). Allelic $\mathrm{R}^{2}$ has a simple interpretation in terms of statistical power, because it measures the loss of power when the most likely imputed genotypes are used in place of the true genotypes for a marker (Browning and Browning, 2009).

\section{Comparison of Accuracy Between Animals With and Without Genotyped Parents}

The difference in concordance rate realized between groups with one or no parents genotyped was determined in the Ayrshire breed. This was done by creating separate validation groups, those with and those without having their sire or maternal grandsire genotyped. It should be noted that all genotyped Ayrshires were males, so there were no genotyped dams. Realized differences in accuracy were then determined. Concordance rates were calculated for each individual 


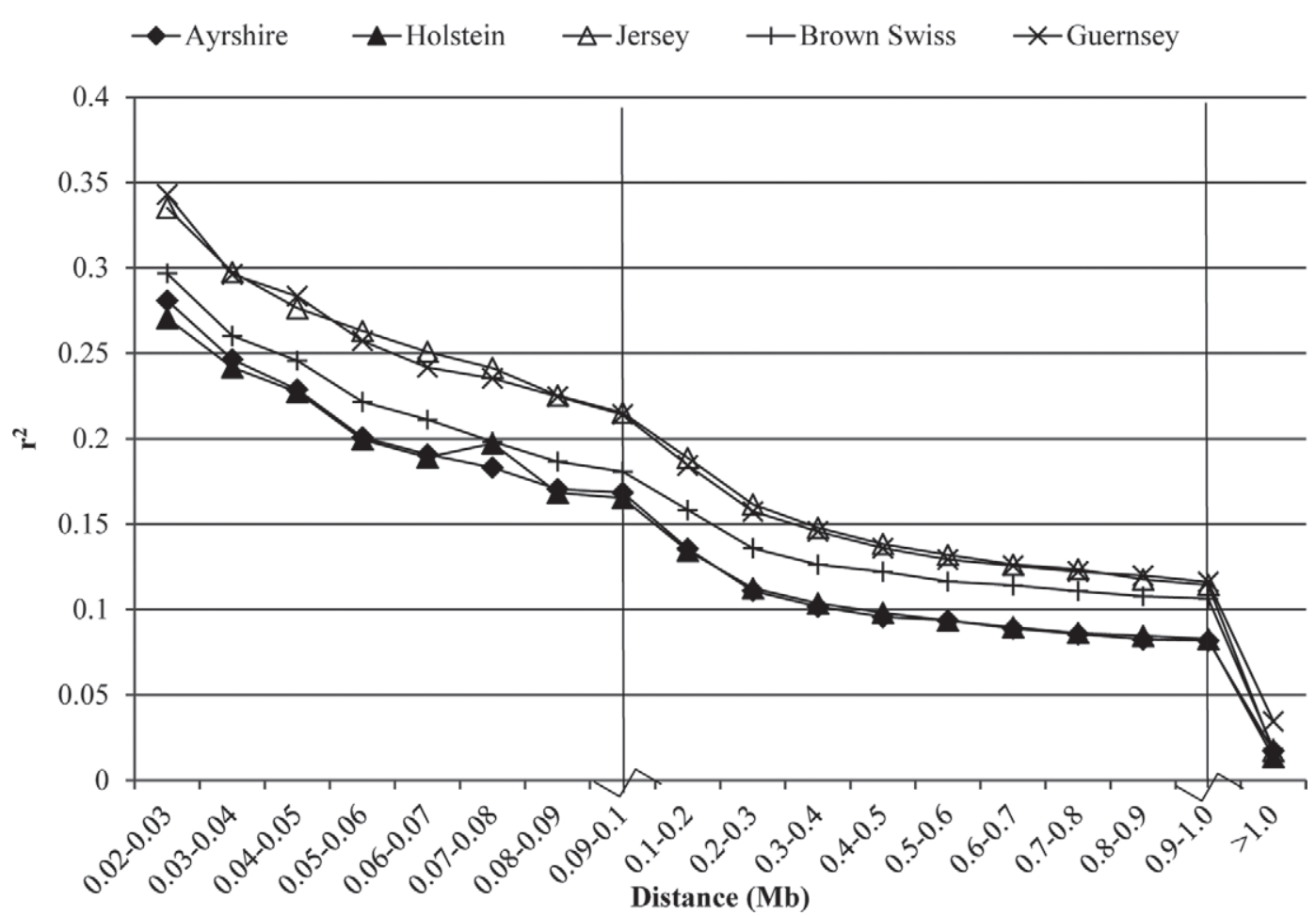

Figure 1. Average $\mathrm{r}^{2}$ values at given distances between markers for 5 breeds using the 50,000 (50K) SNP panel (Illumina Bovine SNP50 BeadChip; Illumina Inc., San Diego, CA).

animal in both groups by calculating the proportion of SNP that matched the original genotype before filtering out SNP not included on the $777 \mathrm{~K}$ panel for that animal, and were used as a new data set for analysis. The ANOVA procedure in SAS (SAS Institute Inc.) was used to determine if there was a significant effect of having a sire genotyped in all scenarios for the Ayrshire breed.

\section{Computing Time}

The amount of time used imputing in each scenario with each algorithm was also monitored. This was done on a per-chromosome basis because a different number of chromosomes were run in parallel in each scenario due to computational requirements.

\section{RESULTS}

\section{Extent of $L D$}

The $\mathrm{r}^{2}$ values for all breeds estimated using the $50 \mathrm{~K}$ SNP chip are presented in Figure 1. The Guernsey and Jersey breeds had the greatest level of LD across all distances; LD in Ayrshires and Holsteins was the lowest at all distances. Trends for all breeds across distances were very similar and linkage decayed at a very similar rate in all breeds. As distances decreased, however, fewer marker pairs were available to be examined.

The $\mathrm{r}^{2}$ values estimated using the $777 \mathrm{~K}$ panel for Ayrshires, Holsteins, and Guernseys are presented in Figure 2. The results showed a large increase in LD as marker distance approached zero. An $\mathrm{r}^{2}$ value of $\sim 0.65$ to 0.7 at a distance of $\sim 0.004 \mathrm{Mb}$ was observed, which is the average distance between markers using the $777 \mathrm{~K}$ panel. If half of this distance was considered the expected maximum distance, on average, between a marker and a QTL, the $\mathrm{r}^{2}$ was $\sim 0.75$ in all 3 breeds. The results at distances similar to those examined on the $50 \mathrm{~K}$ panel were very consistent between the 2 panels.

The $\mathrm{r}^{2}$ values for adjacent SNP were also examined and are presented in Table 1 . The $\mathrm{r}^{2}$ values were taken on a per-chromosome basis, and an overall weighted average based on the number of adjacent pairs on each chromosome was calculated.

\section{Correlation of Linkage Phase}

Consistency of linkage phase, measured as the correlation between signed $r$ values, was assessed between all pairs of breeds using the 50K SNP panel. Results are presented in Table 2 for adjacent SNP pairs and in Figure 3 for different distances between SNP pairs. The 
Table 1. Average squared correlation $\left(\mathrm{r}^{2}\right)$ between adjacent SNP by breed and SNP panel

\begin{tabular}{lcc}
\hline Breed & ${\text { Average } \mathrm{r}^{2}}$ & Distance $^{2}$ \\
\hline $50 \mathrm{~K}$ & & \\
Ayrshire & 0.216 & 0.096 \\
Holstein & 0.215 & 0.082 \\
Jersey & 0.274 & 0.095 \\
Brown Swiss & 0.238 & 0.091 \\
Guernsey & 0.265 & 0.103 \\
$777 \mathrm{~K}$ & & \\
Ayrshire & 0.585 & 0.005 \\
Holstein & 0.589 & 0.005 \\
Guernsey & 0.635 & 0.005
\end{tabular}

${ }^{1} 50 \mathrm{~K}(50,000 \mathrm{SNP})=$ Illumina Bovine SNP50 BeadChip and $777 \mathrm{~K}$ $(777,000 \mathrm{SNP})=$ Illumina BovineHD BeadChip (Illumina Inc., San Diego, CA).

${ }^{2}$ Average distance between adjacent SNP based on number of SNP after filtering for minor allele frequency.

highest correlation was found between Ayrshires and Holsteins. Correlations of all breeds showed a similar pattern across distances with correlations at 0.07 to $0.08 \mathrm{Mb}$ (the average distance between adjacent markers using the $50 \mathrm{~K}$ panel) between 0.46 and 0.57 across breeds. All breed pairs show a strong upward trend until 0.02 Mb (Figure 3).

Consistency of linkage phase was also examined between Ayrshires, Guernseys, and Holsteins using the 777K SNP panel. Results are presented in Table 2 for adjacent SNP pairs and in Figure 4 for different dis-
Table 2. Consistency of linkage phase measured as the average Pearson correlation of signed $r$ value between adjacent SNP by breed pair $^{1}$ and SNP panel ${ }^{2}$

\begin{tabular}{lc}
\hline Breed pair & Correlation \\
\hline $50 \mathrm{~K}$ & \\
AY-HO & 0.57 \\
AY-JE & 0.51 \\
AY-BS & 0.47 \\
AY-GU & 0.49 \\
HO-JE & 0.50 \\
HO-BS & 0.47 \\
HO-GU & 0.48 \\
JE-BS & 0.46 \\
JE-GU & 0.52 \\
BS-GU & 0.46 \\
$777 K$ & \\
AY-HO & 0.95 \\
AY-GU & 0.93 \\
HO-GU & 0.93
\end{tabular}

${ }^{1} \mathrm{AY}=$ Ayrshire, $\mathrm{HO}=$ Holstein, $\mathrm{JE}=$ Jersey, BS $=$ Brown Swiss, GU $=$ Guernsey.

${ }^{2} 50 \mathrm{~K}(50,000 \mathrm{SNP})=$ Illumina Bovine SNP50 BeadChip and $777 \mathrm{~K}$ $(777,000$ SNP) $=$ Illumina BovineHD BeadChip (Illumina Inc., San Diego, CA).

tances between SNP pairs. Similar results were found at the distances examined using the $50 \mathrm{~K}$ panel. When very short distances were considered, very high linkage phase consistency was found, approaching 1, as marker distance approached 0 . Correlations were consistently higher between Ayrshires and Holsteins than either

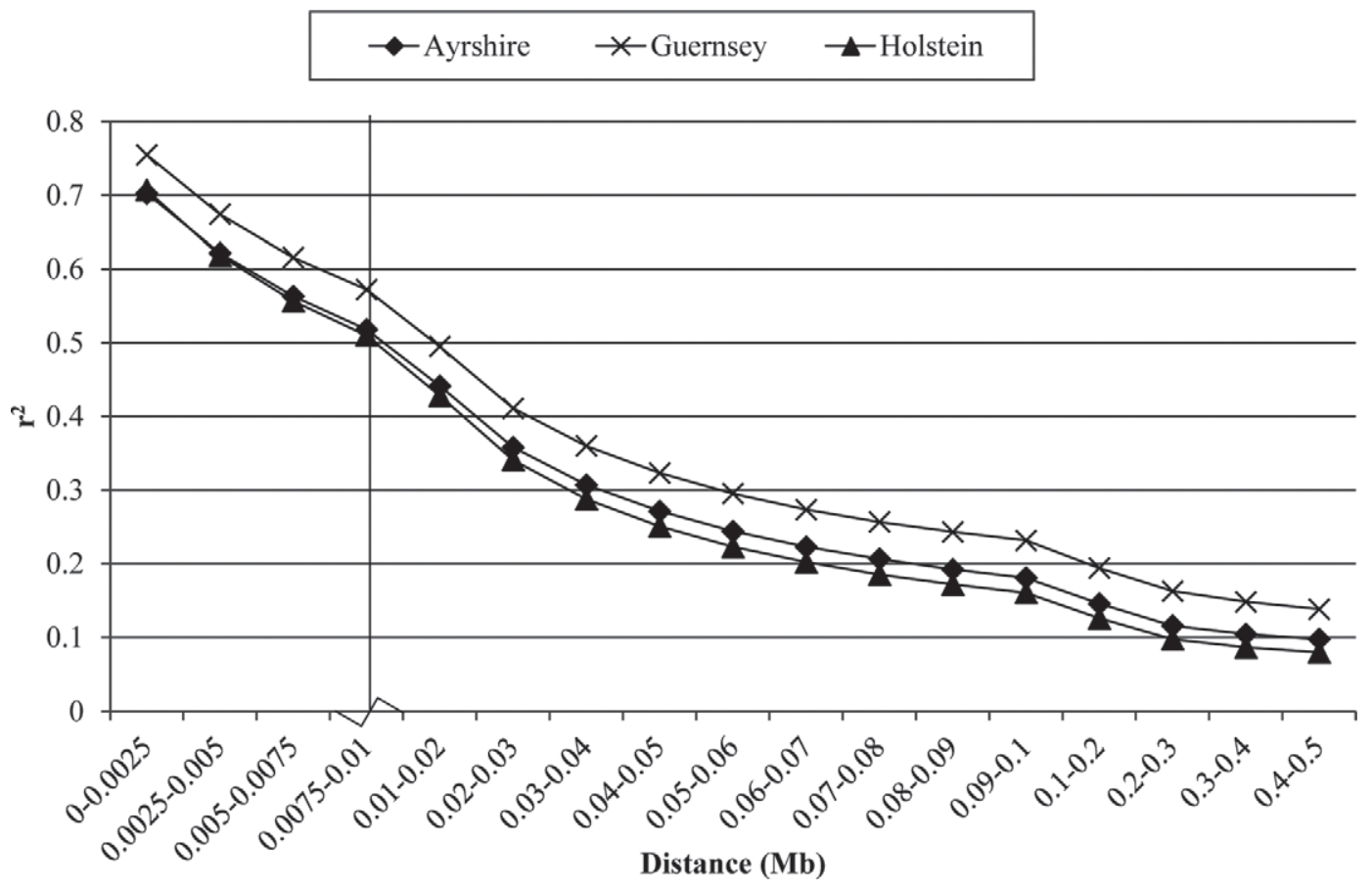

Figure 2. Average $\mathrm{r}^{2}$ values for Ayrshires, Guernseys, and Holsteins at given distances between markers using the 777,000 (777K) SNP panel (Illumina BovineHD BeadChip; Illumina Inc., San Diego, CA). 


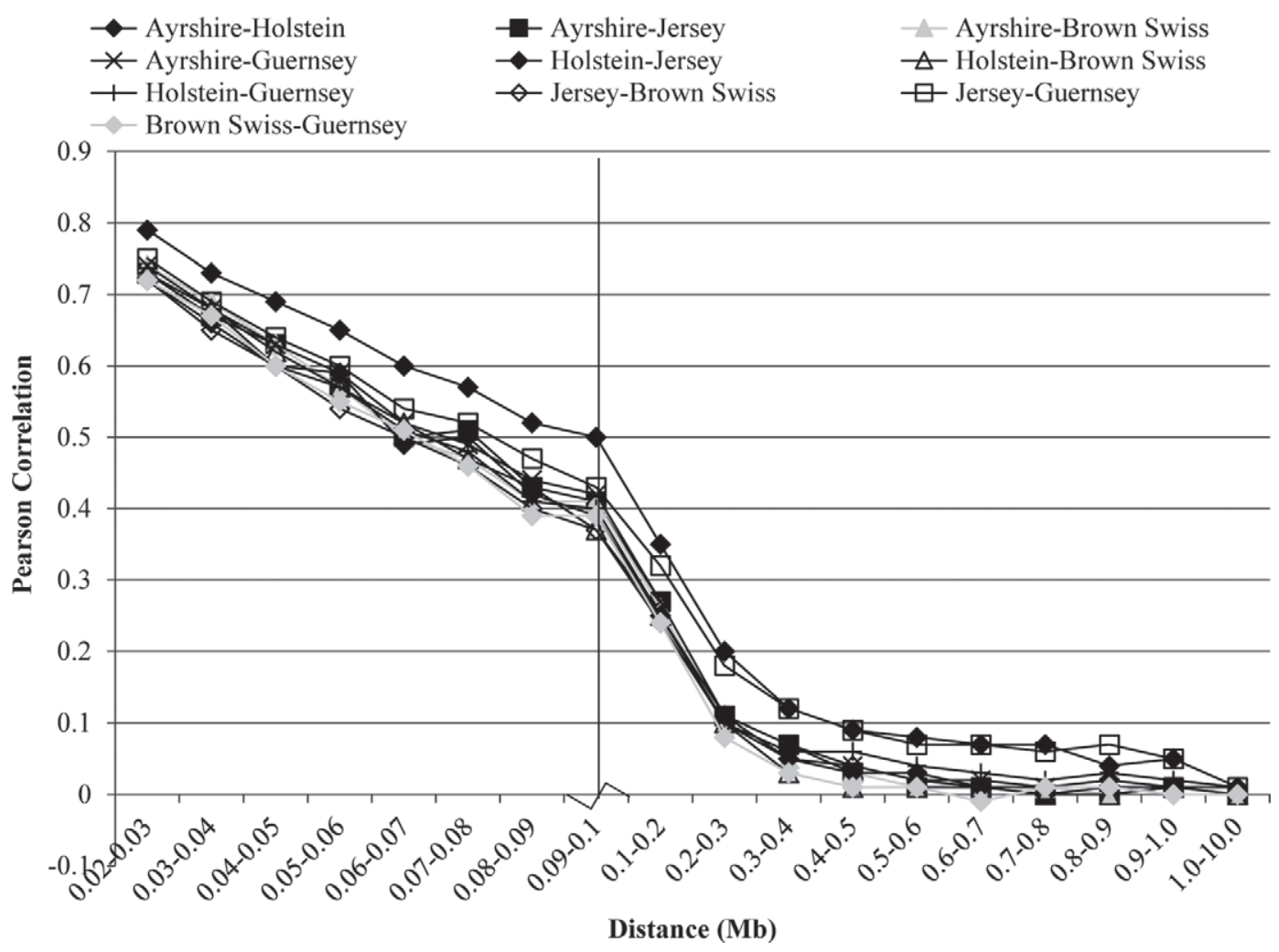

Figure 3. Pearson correlations of signed $\mathrm{r}$ values at given distances for 10 breed pairs using the 50,000 (50K) SNP panel (Illumina Bovine SNP50 BeadChip; Illumina Inc., San Diego, CA).

breed paired with Guernsey. This is consistent with the findings when looking at the $50 \mathrm{~K}$ panel.

\section{Imputation Accuracy}

Concordance rate and allelic $\mathrm{R}^{2}$ displayed a similar pattern across scenarios and imputation algorithms, allelic $\mathrm{R}^{2}$ usually being lower than the concordance rate. The differences were more pronounced in scenarios where the concordance rates were lower.

The difference between population imputation compared with imputation using both family and population information from the $50 \mathrm{~K}$ SNP chip to the $777 \mathrm{~K}$ chip was examined using FImpute for both scenarios (Table 3 ). We detected no significant difference in imputation accuracy between these 2 scenarios; that is, when FImpute was used with or without pedigree information in all 3 breeds (Holsteins, Ayrshires, Guernseys). The algorithm used by FImpute is able to detect parents by finding long-shared haplotypes between reference and imputation sets, so pedigree information has a small effect at moderate low density, such as the $6 \mathrm{~K}$ panel.

The difference between the population-based imputation performed by Beagle and imputation performed by FImpute when pedigree information was omitted (population imputation only) was also evaluated (Table $3)$. We observed very little difference in imputation accuracy between the 2 methods when population-based imputation was carried out on the Holstein population. We did find a difference, however, when the imputation accuracy for Guernsey and Ayrshire was examined, with FImpute outperforming Beagle in both cases. Concordance rate and allelic $\mathrm{R}^{2}$ were higher by $1 \%$ and $3 \%$, respectively, for the Ayrshire breed and by $2 \%$ and $3 \%$, respectively, for the Guernsey breed. As the reference population size decreased, it seemed that FImpute performed better than Beagle. The effect on a per-animal basis was also examined within the Guernsey breed and is presented in Figure 5. FImpute consistently outperformed Beagle on a per-animal basis, and in general, animals that were imputed less accurately by one algorithm were also imputed less accurately by the other algorithm, both in terms of genotype concordance rate (Figure 5) and allelic $\mathrm{R}^{2}$ (not shown).

The use of all breeds as a reference population for imputation using both Beagle and FImpute (without pedigree information) was also assessed (Table 3). For both breeds with smaller reference populations (Ayrshire and Guernsey), an increase in imputation accuracy was observed when information from the other 


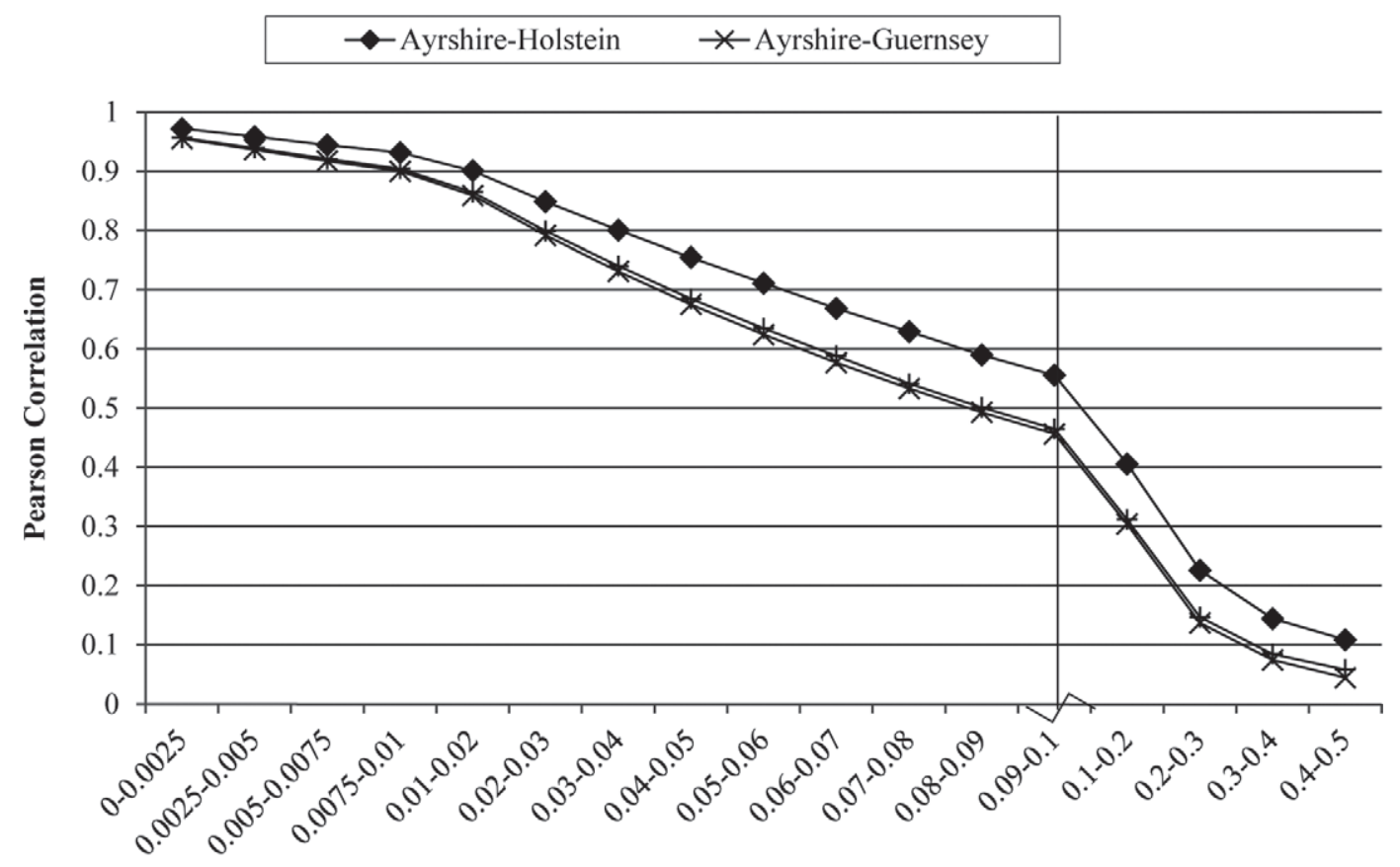

Distance (Mb)

Figure 4. Pearson correlations of signed $\mathrm{r}$ values between Ayrshires, Holsteins, and Guernseys at given distances using the 777,000 (777K) SNP panel (Illumina BovineHD BeadChip; Illumina Inc., San Diego, CA).

Table 3. Accuracy $^{1}$ when imputing from $6,000(6 \mathrm{~K})$ or $50,000(50 \mathrm{~K})$ to $777,000(777 \mathrm{~K})$ SNP for different scenarios using FImpute (Sargolzaei et al., 2011a) or Beagle (Browning and Browning, 2007)

\begin{tabular}{|c|c|c|c|c|}
\hline Breed & Panel $^{2}$ & Scenario $^{3}$ & Concordance rate & Allelic $\mathrm{R}^{2}$ \\
\hline \multirow[t]{5}{*}{ Guernsey } & \multirow[t]{3}{*}{$50 \mathrm{~K}$} & Fam + Pop & 0.970 & 0.949 \\
\hline & & Pop & $0.968(0.951)$ & $0.946(0.915)$ \\
\hline & & Pop (Multi-breed) & $0.981(0.967)$ & $0.971(0.951)$ \\
\hline & \multirow[t]{2}{*}{$6 \mathrm{~K}$} & 1-step & 0.935 & 0.901 \\
\hline & & 1-step (Multi-breed) & 0.918 & 0.872 \\
\hline \multirow[t]{5}{*}{ Ayrshire } & \multirow[t]{3}{*}{$50 \mathrm{~K}$} & Fam+Pop & 0.983 & 0.975 \\
\hline & & Pop & $0.981(0.969)$ & $0.972(0.944)$ \\
\hline & & Pop (Multi-breed) & $0.984(0.978)$ & $0.976(0.968)$ \\
\hline & \multirow[t]{2}{*}{$6 \mathrm{~K}$} & 1-step & 0.956 & 0.935 \\
\hline & & 1-step (Multi-breed) & 0.950 & 0.926 \\
\hline \multirow[t]{8}{*}{ Holstein } & \multirow[t]{3}{*}{$50 \mathrm{~K}$} & Fam+Pop & 0.993 & 0.989 \\
\hline & & Pop & $0.995(0.989)$ & $0.991(0.975)$ \\
\hline & & Pop (Multi-breed) & $0.993(0.993)$ & $0.989(0.989)$ \\
\hline & \multirow[t]{5}{*}{$6 \mathrm{~K}$} & 1-step & 0.977 & 0.965 \\
\hline & & 2-step $(2,000)^{4}$ & 0.981 & 0.971 \\
\hline & & 2 -step $(4,000)^{4}$ & 0.981 & 0.971 \\
\hline & & 2-step $(6,000)^{4}$ & 0.982 & 0.972 \\
\hline & & 1-step (Multi-breed) & 0.975 & 0.962 \\
\hline
\end{tabular}

${ }^{1}$ Accuracy was measured as both concordance rate and allelic $\mathrm{R}^{2}$; accuracy for Beagle is shown in parentheses. ${ }^{2} 6 \mathrm{~K}=$ Illumina BovineLD Genotyping BeadChip, 50K = Illumina Bovine SNP50 BeadChip, and 777K = Illumina BovineHD BeadChip (Illumina Inc., San Diego, CA).

${ }^{3}$ Fam +Pop: both family- and population-based imputation algorithms were used; Pop: pedigree information was excluded and only population-based imputation was carried out; Multi-breed: all 3 breeds in the multibreed reference population instead of single breed; 6K 1-step: Fam+Pop imputation was carried out directly from $6 \mathrm{~K}$ to $777 \mathrm{~K}$; $6 \mathrm{~K}$ 2-step: Fam+Pop imputation was carried out from $6 \mathrm{~K}$ to $50 \mathrm{~K}$ and then from $50 \mathrm{~K}$ to $777 \mathrm{~K}$.

${ }^{4}$ Number of $50 \mathrm{~K}$ genotypes added to the reference population in the first step of 2-step imputation within breed. 


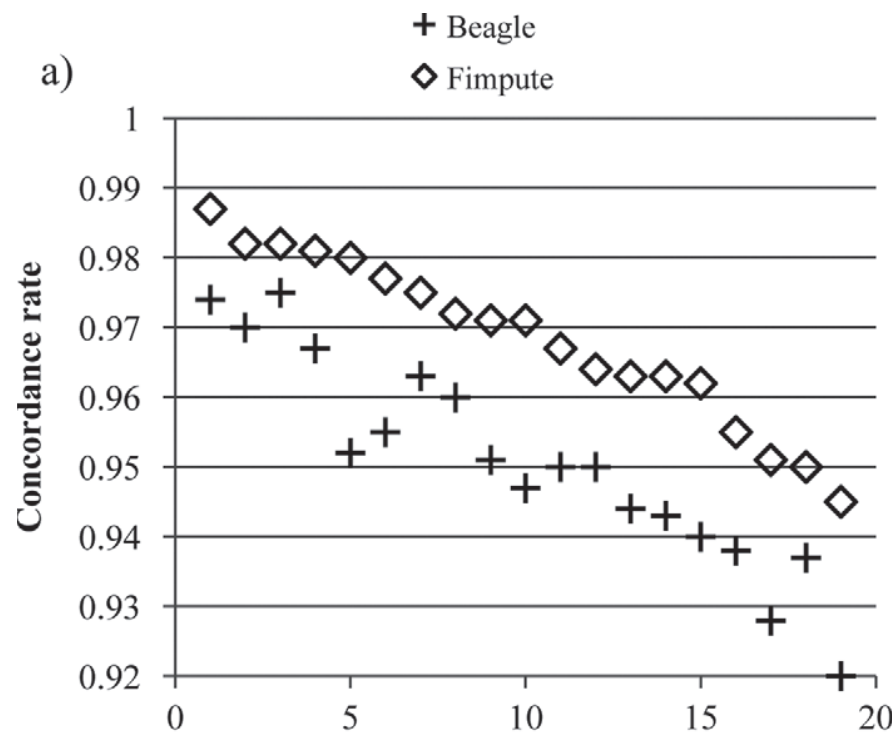

b)

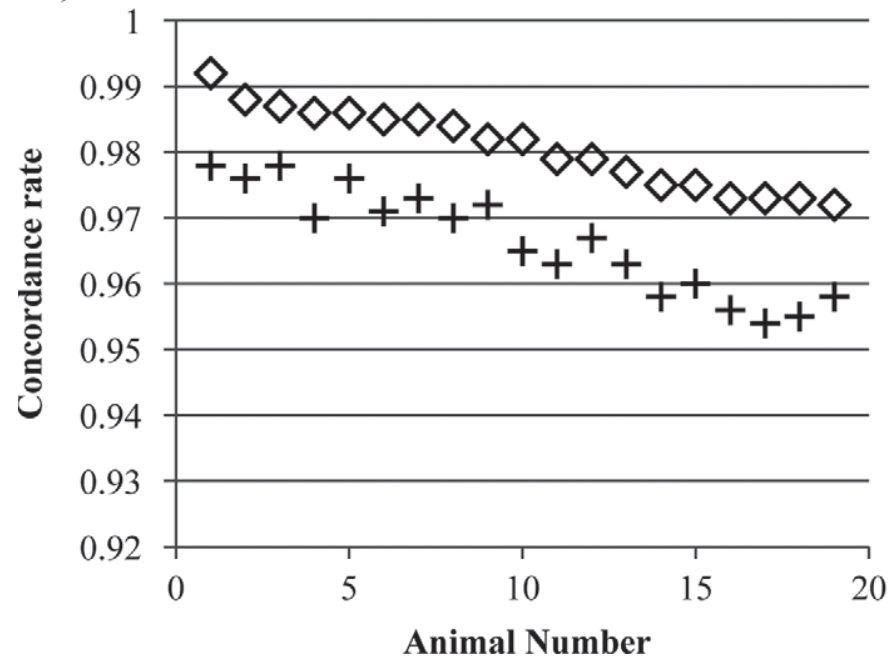

Figure 5. Population imputation accuracy from 50,000 (50K; Illumina Bovine SNP50 BeadChip; Illumina Inc., San Diego, CA) to 777,000 (777K; Illumina BovineHD BeadChip; Illumina Inc.) SNP by animal for Guernseys, using a (a) single-breed or (b) multi-breed reference population.

2 breeds was included, especially when allelic $\mathrm{R}^{2}$ was considered. This difference was greater when imputation was carried out with Beagle. The largest difference was seen for Beagle in the Guernsey population when information from Holstein and Ayrshire were included. Including other breeds led to an increase of more than $1.5 \%$ in concordance rate and $3.5 \%$ increase in allelic $\mathrm{R}^{2}$. FImpute also gained from using information from other breeds, although the difference was smaller. FImpute maintained higher imputation accuracy in all scenarios, even when large gains were seen with Beagle.
Imputation accuracy was also determined when imputing from $6 \mathrm{~K}$ to $777 \mathrm{~K}$ (Table 3 ). Imputation accuracy was generally lower when imputing from $6 \mathrm{~K}$ to the $777 \mathrm{~K}$ panel than from $50 \mathrm{~K}$ to $777 \mathrm{~K}$, especially in breeds with smaller reference populations than Holsteins. The imputation accuracy was relatively high in Holstein with a concordance rate of 0.977 and allelic $\mathrm{R}^{2}$ of 0.971 , but was substantially lower for Guernsey (0.935 and 0.901) and Ayrshire (0.956 and 0.935). Imputing in 2 steps yielded a higher accuracy than when imputation was carried out directly from $6 \mathrm{~K}$ to the high-density platform in the Holstein breed (Table 3). Adding 50K reference genotypes in the first step of 2-step imputation was beneficial; however, little difference was seen when more than 2,000 genotypes were added.

Imputation from $6 \mathrm{~K}$ to $777 \mathrm{~K}$ using a multi-breed reference population was detrimental compared with imputation carried out with a single-breed reference population for the breeds with low to moderate reference sizes. Concordance rate and allelic $\mathrm{R}^{2}$ were decreased by $1.5 \%$ and $3 \%$ in Guernsey and by $0.5 \%$ and $1 \%$ in Ayrshire, respectively.

\section{Assessing the Effect of Family Information}

Concordance rates were measured on a per-animal basis and used to determine if there was a significant effect of having or not having a genotyped sire. Table 4 presents the number of imputed animals with genotyped ancestors in the reference population for each breed. Table 5 shows the results of this analysis for the Ayrshire breed. Mean concordance rate is presented, as well as the difference between animals with or without their sire in the genotyped reference population. In addition, Table 5 presents the corresponding $P$-values from the ANOVA used to determine if a significant effect of having sire genotyped existed. Data were first transformed using the log score of 1 minus the concordance rate, to normalize the data to meet the normality assumptions of ANOVA. In all of the scenarios, the $P$-value was $\leq 0.05$, and the effect of having a sire in the genotyped reference population was deemed significant.

Table 4. Number of imputation animals with genotyped ancestors in the reference population

\begin{tabular}{lccc}
\hline Genotyped parent(s) & $\begin{array}{c}\text { Holstein } \\
(\mathrm{n}=223)\end{array}$ & $\begin{array}{c}\text { Ayrshire } \\
(\mathrm{n}=164)\end{array}$ & $\begin{array}{c}\text { Guernsey } \\
(\mathrm{n}=19)\end{array}$ \\
\hline Sire & 83 & 84 & 0 \\
Dam & 25 & 0 & 0 \\
Maternal grandsire $^{1}$ & 21 & 13 & 0 \\
Paternal grandsire $^{1}$ & 60 & 54 & 0
\end{tabular}

${ }^{1}$ All animals with genotyped maternal grandsire and paternal grandsire also have sire genotyped. 
Table 5. Effect of having a genotyped parent (sire) or maternal grandparent (MGS) in the reference population for different scenarios ${ }^{1}$ in the Ayrshire breed with $P$-values from ANOVA comparing accuracy of imputation from $6,000(6 \mathrm{~K})$ or $50,000(50 \mathrm{~K})$ to $777,000(777 \mathrm{~K}) \mathrm{SNP}^{2}$ between animals with and without genotyped sires

\begin{tabular}{llccrr}
\hline Algorithm $^{3}$ & Scenario & $\begin{array}{c}\text { Sire genotyped } \\
(\mathrm{n}=97) \mathrm{CR}^{4}\end{array}$ & $\begin{array}{c}\text { Sire not genotyped } \\
(\mathrm{n}=67) \mathrm{CR}\end{array}$ & $\Delta \mathrm{CR}$ & $P$-value \\
\hline FImpute & 50K Fam+Pop & 0.987 & 0.980 & 0.007 & $<0.001$ \\
& 50K Pop & 0.984 & 0.979 & 0.005 & 0.031 \\
Beagle & 50K Pop & 0.972 & 0.964 & 0.008 & 0.005 \\
FImpute & 50K Pop (Multi-breed) & 0.987 & 0.982 & 0.005 & 0.025 \\
Beagle & 50K Pop (Multi-breed) & 0.981 & 0.976 & 0.005 & 0.050 \\
FImpute & 6K 1-step & 0.962 & 0.945 & 0.017 & $<0.001$ \\
& 6K 1-step (Multi-breed) & 0.962 & 0.941 & 0.021 & $<0.001$ \\
\hline
\end{tabular}

${ }^{1}$ Fam+Pop: both family- and population-based imputation algorithms were used; Pop: pedigree information was excluded and only population-based imputation was carried out; Multi-breed: all 3 breeds in the multibreed reference population instead of single breed; 6K 1-step: Fam+Pop imputation was carried out directly from $6 \mathrm{~K}$ to $777 \mathrm{~K}$.

${ }^{2} 6 \mathrm{~K}=$ Illumina BovineLD Genotyping BeadChip, 50K = Illumina Bovine SNP50 BeadChip, and 777K = Illumina BovineHD BeadChip (Illumina Inc., San Diego, CA).

${ }^{3}$ FImpute (Sargolzaei et al., 2011a); Beagle (Browning and Browning, 2007).

${ }^{4}$ Concordance rate (proportion of correct calls within imputed SNP); $\Delta=$ change in value.

\section{Computing Time}

A subset of results for overall computing time and computing time per chromosome for all imputation scenarios are presented in Figure 6; this subset is representative of all scenarios. The largest differences in computing were seen between Beagle and FImpute, with FImpute being 10 to 30 times more computationally efficient in all comparable scenarios. We also noted a difference in computing time within the FImpute imputation program when pedigree information was included or omitted. A slight increase in computational efficiency was seen when pedigree information was excluded.

\section{DISCUSSION}

\section{$L D$}

The level of LD is a major factor in the ability to implement genomic selection, as populations with limited LD will require many more markers to accurately predict breeding values. The levels of LD in Holsteins found in this study were consistent with that found in other studies (Sargolzaei et al., 2008; Qanbari et al., 2010; Zhou et al., 2013). The level of LD in all other breeds was found to be higher at all measured distances. This is consistent with the smaller effective population sizes found in colored dairy breeds relative to Holsteins by Stachowicz et al. (2011).

Measures of LD using the $777 \mathrm{~K}$ panel at average adjacent marker distances $(\sim 0.004 \mathrm{Mb})$ were high enough in all breeds to suggest that association studies as well as genomic selection would be possible given a large enough training population; LD values $\left(\mathrm{r}^{2}\right)$ exceeded
0.6 at these distances. Adjacent marker $\mathrm{r}^{2}$ values were $0.585,0.635$, and 0.588 for Ayrshires, Guernseys, and Holsteins respectively. Assuming all QTL are, at most, at half of the distance between adjacent markers, $r^{2}$ values were even higher and exceeded 0.6 for all breeds.

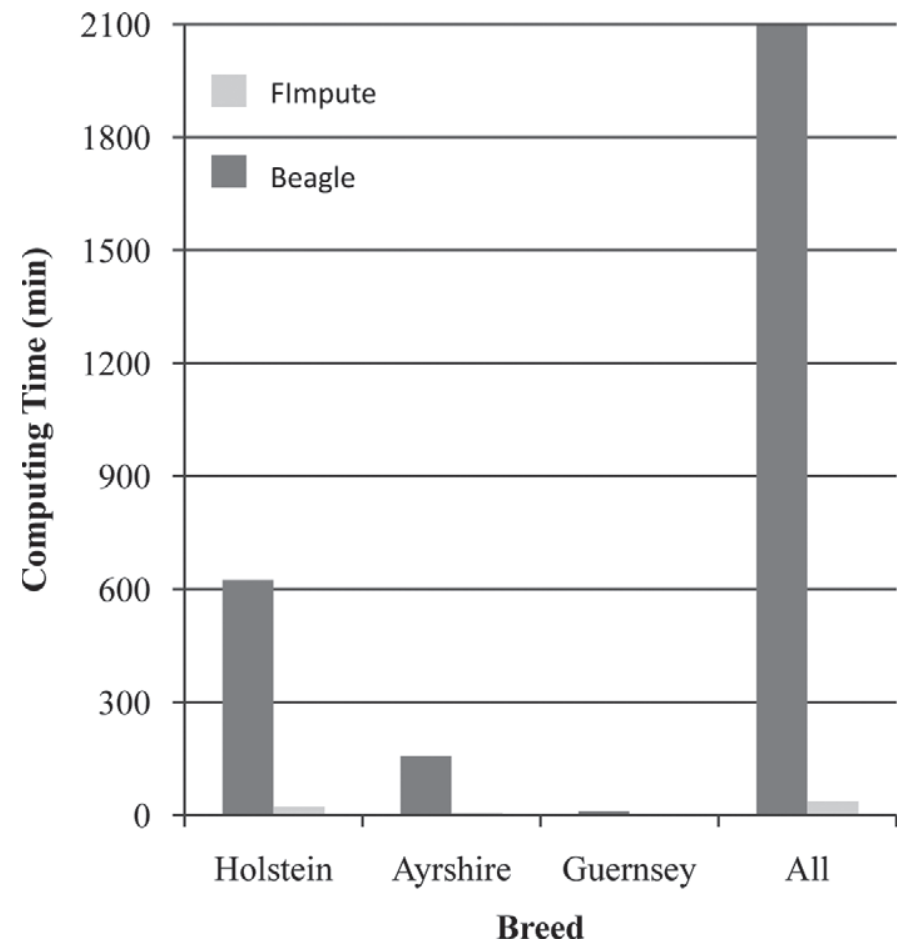

Figure 6. Per-chromosome computing time for each breed for population imputation from 50,000 (50K; Illumina Bovine SNP50 BeadChip; Illumina Inc., San Diego, CA) to 777,000 (777K; Illumina BovineHD BeadChip; Illumina Inc.) SNP using a single-breed or multi-breed (All) reference population. 
Results were consistent between the $50 \mathrm{~K}$ and $777 \mathrm{~K}$ panels at the same distances for all breed pairs.

\section{Consistency of Phase}

The breed pair of Holstein and Ayrshire showed the highest phase correlation at all distances on both SNP panels. This leads to the hypothesis that these 2 breeds diverged more recently than other breed pairs or have undergone a greater degree of admixture since the breeds diverged historically. As phase correlation was higher for this breed pair at both short and long distances between marker pairs, a combination of these factors likely led to a greater correlation of phase between Holstein and Ayrshire populations.

When short distances on the $777 \mathrm{~K}$ panel were examined, a strong correlation of phase across breeds was found, $>0.90$ in all breed pairs. With high correlations, such as the ones found in this study, one can assume that markers and QTL phases are highly associated across breeds. Using a large number of markers, Rolf et al. (2010) found that when the high-density panel was used on several beef breeds, 1 SNP on BTA29 was found to be significantly associated with beef tenderness across breeds, which was not found using the $50 \mathrm{~K}$ panel. Assuming that many marker QTL relationships such as this exist across the genome, we can speculate that implementation of genotyping with the high-density panel will allow for across-breed genomic selection to be more effective, as was seen to a degree in Erbe et al. (2012) when certain models were employed. Although gains in accuracy of genomic selection using the $777 \mathrm{~K}$ panel have been modest to date, both within and across breeds (Erbe et al., 2012; Su et al., 2012), consistent gametic phase across breeds at short distances demonstrates the existence of conserved segments of the genome among the dairy breeds. New models may have to be developed to fully exploit these conserved genome segments and implement across-breed genomic selection.

\section{Imputation Accuracy}

The relationship between concordance rate and allelic $\mathrm{R}^{2}$ was consistent across scenarios, where allelic $\mathrm{R}^{2}$ was lower for all scenarios. The differences were more pronounced for lower accuracies. Alleles with low minor allele frequency are more difficult to impute, and $\mathrm{R}^{2}$ better captures the difference between imputation accuracy and correct filling by chance than concordance rate, which usually translates to lower values for $\mathrm{R}^{2}$ compared with concordance rates (e.g., Howie et al., 2009; Ma et al., 2013).
Family-based imputation algorithms have been shown to be extremely effective in dairy cattle populations with a high level of pedigree information and a large reference population (Zhang and Druet, 2010; Sargolzaei et al., 2011b; Mulder et al., 2012). The results presented in this study, however, show that a population-based imputation algorithm can be just as accurate in all sizes of reference population when imputation animals are genotyped with a sufficiently dense panel. The imputation animals within the Guernsey breed had no genotyped ancestors, whereas Holsteins and Ayrshires had some ancestors genotyped both maternally and paternally. We found no difference in accuracy between combined family- and population-based and population-based algorithms in any case; however, this should be the case because the population-based algorithm in FImpute is still able to detect ancestors without pedigree information based on long-conserved segments of haplotypes. Results on a per-animal basis show that FImpute consistently outperforms Beagle when population size is small and family information is limited. Previous studies showing an advantage of combined family- and population-based imputation methods have focused on imputation from either the $3 \mathrm{~K}$ panel or the $6 \mathrm{~K}$ panel to the $50 \mathrm{~K}$ SNP chip. For lower densities, such as the 3K SNP panel, pedigree information has a greater effect, increasing imputation accuracy substantially (Sargolzaei et al., 2011b). When imputing from $50 \mathrm{~K}$ to $777 \mathrm{~K}$, shorter segments of haplotype conserved over many generations can be found compared with the lower density panels and thus can be accurately imputed using only a population-based algorithm without knowing any pedigree information.

The Beagle imputation method has been used extensively in human and livestock genome studies. In this study, Beagle was compared with the population algorithm in FImpute, which assumes some level of relatedness between all animals. FImpute performed more effectively using both metrics of imputation accuracy in most scenarios, especially those in which a smaller reference population was available. Beagle, however, had a greater gain from multi-breed imputation. Beagle considers all animals to be unrelated. Although animals across breeds are generally unrelated, short segments of haplotype are conserved between breeds from before those breeds diverged, which Beagle captures and uses for imputation. FImpute searches for the largest possible consistent haplotypes and iteratively moves to smaller and smaller ones if no consistent haplotype is found in the reference population. Thus, it can move toward looking at very short haplotypes, searching for haplotypes from a common ancestor many generations ago, perhaps before breeds had diverged. This explains 
the small gain still realized by the FImpute software when more than one breed was included in the reference population.

In an effort to grow the reference population for smaller breeds in a fast and economically viable manner, we examined the accuracy of imputing from the $6 \mathrm{~K}$ panel directly to the $777 \mathrm{~K}$ panel. The concordance rate ranged from 0.935 to 0.977 across the 3 breeds; however, for Guernseys and Ayrshires (the breeds with greater need of larger reference populations), concordance rates were 0.935 and 0.956 , respectively, with corresponding allelic $\mathrm{R}^{2}$ of 0.901 and 0.935 . These accuracies are comparable with many population-based imputation accuracies from $3 \mathrm{~K}$ to $50 \mathrm{~K}$, reported by Daetwyler et al. (2011), in which only very modest decreases in accuracy of genomic breeding values were found using these imputed genotypes. Thus, imputation from $6 \mathrm{~K}$ to $777 \mathrm{~K}$ may be feasible in breeds with smaller reference populations and will become increasingly useful as the high-density reference set grows in these populations. Multi-breed imputation was also examined, as consistent phase across breeds should lead to gains in imputation accuracies for breeds with small reference populations. Gains in accuracy were seen in Guernsey and Ayrshire breeds, leading to the conclusion that imputation can be carried out jointly for these breeds from $50 \mathrm{~K}$ to $777 \mathrm{~K}$, where consistency of gametic phase is high. Imputation from a sparser $6 \mathrm{~K}$ panel to a highdensity panel was slightly detrimental compared with imputation carried out within breed for breeds with low to moderate reference sizes, meaning that imputation from $6 \mathrm{~K}$ is best carried out within breed at this density. Gautier et al. (2007) showed that significant consistency of phase is only present at distances less than $0.1 \mathrm{~kb}$. Average marker pair distance on the $6 \mathrm{~K}$ panel is much longer $(\sim 0.5 \mathrm{~kb})$. This leads to a lack of conserved haplotypes between breeds being detected, and very little useful information can be captured from animals outside a breed, as haplotypes are not consistently phased, and SNP will not have consistent associations with QTL. The accuracy of imputation carried out in 1 or 2 steps using the $6 \mathrm{~K}$ panel was also examined in Holsteins. Imputing in 2 steps showed a slight increase in accuracy over imputing directly from $6 \mathrm{~K}$ to $777 \mathrm{~K}$, by adding $2,00050 \mathrm{~K}$ genotypes in the reference population for the first step of the 2-step procedure. Subsequently, adding more $50 \mathrm{~K}$ genotypes to the reference population had a very small effect on imputation accuracy. This, however, comes at the expense of computing time as a larger reference population will result in more haplotypes to be searched by the imputation algorithm. For routine imputation, a reference population of finite size should be constructed to best represent the haplotypes present in the population, while being limited in size to increase computational efficiency.

The allelic $\mathrm{R}^{2}$ in Table 3 give an indication of the loss of power when the most likely imputed genotypes are used in place of the true SNP genotypes in association analyses and genomic predictions. The level of LD between 2 imputed SNP will be a direct function of the allelic $\mathrm{R}^{2}$ and $\mathrm{r}^{2}$ calculated using the true SNP genotypes.

\section{Computing Time}

One important consideration when evaluating the application of imputation to routine genomic evaluations is computing time. Computing time for imputation is determined by the reference population size, the number of animals to impute, and the density of the highand low-density marker panels to which the imputation algorithm is being applied. Imputation carried out with FImpute was more accurate and 10 to 30 times more efficient compared with Beagle for identical scenarios. FImpute is more efficient at capturing long segments consistently between reference and imputation animals, which fills in large portions of the genome, leaving fewer haplotypes to be filled in by considering allele and haplotype frequencies.

\section{Implications}

With a significant increase in $\mathrm{r}^{2}$ values due to a dense SNP panel, GEBV accuracy can be increased with the same number of training animals. By studying the consistency of linkage phase between breeds, GEBV accuracy may be increased by pooling breeds into a common reference population for training of marker effects. The results of this study show strong correlations at close distances using the 777K SNP panel. de Roos et al. (2008) concluded that the accuracy of genomic selection could be increased by combining breeds, if all breeds for which GEBV were to be calculated were included in the reference population. Using a higher density marker panel should also allow genomic predictions to be more accurate over successive generations.

The results of the imputation component of this study look promising for application in genomic selection. Using the high-density marker panel should allow for more accurate genomic selection in breeds with smaller reference populations for the training of SNP effects by using a multi-breed reference set. Daetwyler et al. (2011) found little decrease in accuracy of genomic breeding values from imputed marker panels with an average genotype concordance rate of $\sim 92 \%$. In all situations when the low-density panel was the 
$50 \mathrm{~K}$ panel, a method existed to impute with greater than this level of accuracy for all scenarios studied. An accurate GEBV could be generated for every animal in this study, given a sufficiently large training population for estimating SNP effects. Further studies need to be completed to determine the loss in accuracy of GEBV when the population for training SNP effects is largely composed of imputed animals.

Having a sire in the genotyped reference population increased accuracy of imputation significantly; this gives valuable insight about which animals should be genotyped with the high-density panels in the future to gain the most from large-scale imputation for genomic selection. Key ancestors to the general population should be continually genotyped in an effort to most accurately impute the greatest number of animals. This includes continuing to genotype key proven sires, as they will have highest degree of relatedness to the entire population and thus will lead to the greatest gain in accuracy of imputation and, subsequently, in accuracy of genomic selection per se.

\section{CONCLUSIONS}

Measures of linkage disequilibrium $\left(\mathrm{r}^{2}\right)$ were high, exceeding 0.55 at distances corresponding to adjacent SNP in the $777 \mathrm{~K}$ panel for all breeds. In addition, gametic phase was highly correlated $(>0.95)$ between breeds for adjacent markers in the $777 \mathrm{~K}$ panel. This could allow the use of a multi-breed reference population to increase accuracy of genomic prediction in breeds that have a small reference population of genotyped animals, such as Ayrshire and Guernsey, although new genomic prediction methodology will likely need to be developed for across-breed selection. Imputation results indicate that imputation from the 50K SNP panel to the $777 \mathrm{~K}$ SNP panel can be carried out with sufficient accuracy to potentially allow the genotypes to be included in genomic prediction of dairy breeds with small reference population size. Studies on GEBV accuracies using imputed genotypes are, therefore, warranted.

\section{ACKNOWLEDGMENTS}

This study was funded by a Collaborative Research and Development Grant from the Natural Sciences and Engineering Research Council of Canada (NSERC, Ottawa, ON, Canada) in collaboration with the DairyGen Council of the Canadian Dairy Network (CDN, Guelph, ON, Canada).

\section{REFERENCES}

Ardlie, K. G., L. Kruglyak, and M. Seielstad. 2002. Patterns of linkage disequilibrium in the human genome. Nat. Rev. Genet. 3:299-309.
Brito, F. V., J. Braccini, M. Sargolzaei, J. Cobuci, and F. S. Schenkel. 2011. Accuracy of genomic selection in simulated populations mimicking the extent of linkage disequilibrium in beef cattle. BMC Genet. 12:80-89.

Browning, B. L., and S. R. Browning. 2009. A unified approach to genotype imputation and haplotype-phase inference for large data sets of trios and unrelated individuals. Am. J. Hum. Genet. $84: 210-223$.

Browning, S. R., and B. L. Browning. 2007. Rapid and accurate haplotype phasing and missing-data inference for whole-genome association studies by use of localized haplotype clustering. Am. J. Hum. Genet. 81:1084-1097.

Calus, M. P. L., T. H. E. Meuwissen, A. P. W. de Roos, and R F. Veerkamp. 2008. Accuracy of genomic selection using different methods to define haplotypes. Genetics 178:553-561.

Daetwyler, H. D., G. R. Wiggans, B. J. Hayes, J. A. Woolliams, and M. E. Goddard. 2011. Imputation of missing genotypes from sparse to high density using long-range phasing. Genetics 189:317-327.

de Roos, A. P. W., B. J. Hayes, R. J. Spelman, and M. E. Goddard. 2008. Linkage disequilibrium and persistence of phase in HolsteinFriesian, Jersey and Angus cattle. Genetics 179:1503-1512.

Erbe, M., B. J. Hayes, L. K. Matumalli, S. Goswami, P. J. Bowman, C. M. Reich, B. A. Mason, and M. E. Goddard. 2012. Improving accuracy of genomic predictions within and between dairy cattle breeds with imputed high-density single nucleotide polymorphism panels. J. Dairy Sci. 95:4114-4129.

Farnir, F., W. Coppieters, J. J. Arranz, P. Berzi, N. Cambisano, B. Grisart, L. Karim, F. Marcq, L. Moreau, M. Mni, C. Nezer, P. Simon, P. Vanmanshoven, D. Wagenaar, and M. Georges. 2000. Extensive genome-wide linkage disequilibrium in cattle. Genome Res. 10:220-227.

Gautier, M., T. Faraut, K. Moazami-Goudarzi, V. Navratil, M. Foglio, C. Grohs, A. Boland, J. Garnier, D. Boichard, G. M. Lathrop, I G. Gut, and A. Eggen. 2007. Genetic and haplotypic structure in 14 European and African cattle breeds. Genetics 177:1059-1070.

Hayes, B. J., P. J. Bowman, A. J. Chamberlain, and M. E. Goddard. 2009. Invited review: Genomic selection in dairy cattle: Progress and challenges. J. Dairy Sci. 92:433-443.

Howie, B. N., P. Donnelly, and J. Marchini. 2009. A flexible and accurate genotype imputation method for the next generation of genome-wide association studies. PLoS Genet. 5:e1000529.

Liu, Z., F. R. Seefried, F. Reinhardt, S. Rensing, G. Thaller, and R. Reents. 2011. Impacts of both reference population size and inclusion of a residual polygenic effect on the accuracy of genomic prediction. Genet. Sel. Evol. 43:19.

Luan, T., J. A. Woolliams, S. Lien, M. Kent, M. Svendsen, and T. H. E. Meuwissen. 2009. The accuracy of genomic selection in Norwegian Red cattle assessed by cross-validation. Genetics 183:1119-1126.

Lynch, M., and B. Walsh. 1998. Estimation of gametic phase disequilibrium in genetics and analysis. Pages 98-99 in Genetics and Analysis of Quantitative Traits. Sinauer Associates Inc., Sunderland, MA.

Ma, P., R. F. Brøndum, Q. Zhang, M. S. Lund, and G. Su. 2013. Comparison of different methods for imputing genome-wide marker genotypes in Swedish and Finnish Red Cattle. J. Dairy Sci. 96:4666-4677.

Meuwissen, T. H., B. J. Hayes, and M. Goddard. 2001. Prediction of total genetic value using genome-wide dense marker maps. Genetics 157:1819-1829.

Mulder, H. A., M. P. L. Calus, T. Druet, and C. Schrooten. 2012. Imputation of genotypes with low-density chips and its effect on reliability of direct genomic values in Dutch Holstein cattle. J. Dairy Sci. 95:876-889.

Pryce, J. E., B. Gredler, S. Bolormaa, P. J. Bowman, C. Egger-Danner, C. Fuerst, R. Emmerling, J. Sölkner, M. E. Goddard, and B. J. Hayes. 2011. Short communication: Genomic selection using a multi-breed, across-country reference population. J. Dairy Sci. 94:2625-2630.

Qanbari, S., E. C. G. Pimentel, J. Tetens, G. Thaller, P. Lichter, A. R. Sharifi, and H. Simianer. 2010. The pattern of linkage disequilibrium in German Holstein cattle. Anim. Genet. 41:346-356. 
Rolf, M. M., S. D. McKay, M. C. Mcclure, J. E. Decker, T. M. Taxis, R. H. Chapple, D. A. Vasco, S. J. Gregg, J. W. Kim, R. D. Schnabel, and J. F. Taylor. 2010. How the next generation of genetic technologies will impact beef cattle selection. Pages 46-56 in Beef Improvement Fed. Res. Symp. Annu. Mtg., Columbia, MO. Angus Productions Inc., St. Joseph, MO.

SargolzaeiM.ChesnaisJ. P.SchenkelF. S. 2011a. FImpute-An efficient imputation algorithm for dairy cattle populations. J. Dairy Sci. 94(E-Suppl. 1):421. (Abstr.)

Sargolzaei, M., J. P. Chesnais, and F. S. Schenkel. 2011b. Accuracy of Imputed 50K Genotypes from 3K and 6K Chips Using FImpute Version 2. Canadian Dairy Network Open Industry Session, October 12, 2011, Guelph, Ontario. Accessed May 1, 2013. http://cgil. uoguelph.ca/dcbgc/Agenda1109/DCBGC1109_msargol.pdf.

Sargolzaei, M., F. S. Schenkel, G. B. Jansen, and L. R. Schaeffer. 2008. Extent of linkage disequilibrium in Holstein cattle in North America. J. Dairy Sci. 91:2106-2117.
Stachowicz, K., M. Sargolzaei, F. Miglior, and F. S. Schenkel. 2011. Rates of inbreeding and genetic diversity in Canadian Holstein and Jersey cattle. J. Dairy Sci. 94:5160-5175.

Su, G., R. F. Brøndum, P. Ma, B. Guldbrandtsen, G. P. Aamand, and M. S. Lund. 2012. Comparison of genomic predictions using medium-density $(\sim 54,000)$ and high-density $(\sim 777,000)$ single nucleotide polymorphism marker panels in Nordic Holstein and Red Dairy Cattle populations. J. Dairy Sci. 95:4657-4665.

Zhang, Z., and T. Druet. 2010. Marker imputation with low-density marker panels in Dutch Holstein cattle. J. Dairy Sci. 93:54875494.

Zhou, L., X. Ding, Q. Zhang, Y. Wang, M. S. Lund, and G. Su. 2013. Consistency of linkage disequilibrium between Chinese and Nordic Holsteins and genomic prediction for Chinese Holsteins using a joint reference population. Genet. Sel. Evol. 45:7. 